\title{
CONTROLLING PENETRATION ZONE FORMATION IN ARC SURFACING
}

\author{
V.P. IVANOV and E.V. LAVROVA \\ Priazovsky State Technical University \\ 7 Universitetskaya Str., 87500, Mariupol, Ukraine. E-mail: pstu.edu.oitzv@gmail.com
}

\begin{abstract}
Influence of surfacing mode parameters on penetration zone formation was studied, and methods of controlling and stabilizing the process of weld pool formation were determined. Models of penetration zone formation for stationary and moving electrodes were developed. Calculated dependencies were determined by statistical processing of the results of measuring penetration zone parameters in macrosections of samples produced by submerged-arc surfacing for different electrode diameters and applied feed rates (current), depending on arcing time and arc movement speed. Obtained mathematical models of penetration zone formation in submerged-arc surfacing were used to develop software and control system of surfacing equipment. 8 Ref., 1 Table, 9 Figures.
\end{abstract}

Keywords : weld pool, penetration zone, mathematical model, control of weld pool formation process

Modern production requires development of adequate models of processes occurring in the weld pool, and CAD-CAE-CAM tools.

Controlling weld pool formation allows ensuring the quality of (pad) weld at changing of technological process parameters. At present, technologies of welding and surfacing both with wire $[1,2]$, and strip electrodes $[3,4]$ have been developed, in which higher effectiveness is achieved not by stabilization of mode parameters, but by their variation by certain functional dependencies.

At deposition of reconditioning and protective layers attention is focused on deposited layer formation, while the main function of penetration zone is reliable adhesion of the layers for preservation of overall performance of surfaced part or tool. Increase of penetration zone leads not only to increase of surfacing cost, but also to lowering of deposited payer performance, because of its heating and increase of the proportion of base metal in the deposited metal.

Dimensions of surfacing and penetration zones are directly proportional to electrode feed rate at specified type and material of the electrode, as well as to its melting conditions [5, 6]. Control of these zones formation requires clear understanding of the mechanism and sequence of their formation and their mutual influence. Running of these processes in the liquid phase necessitates determination of time and rate characteristics of melting front propagation as the base for construction of mathematical models of weld formation.

The aim of the work is studying the process of penetration zone formation in submerged-arc surfacing, determination of effective methods of controlling this zone parameters, as well as development of tech-

(C) V.P. IVANOV and E.V. LAVROVA, 2016 nology, equipment and control systems for realization of such technology.

In keeping with the defined task, dynamics of penetration zone formation in surfacing low-carbon steel plates $(350 \times 240 \times 30 \mathrm{~mm})$ with stationary electrode using AN-60 and AN-348A fluxes on stainless steel substrate was studied. Surfacing was conducted with wire electrode Sv-08A (used wire diameters and surfacing mode parameters are given in the Table). Arcing time was recorded using a timer (from 5 to 10 samples in each studied interval). After surfacing the samples were cut with abrasive wheel so that the cut line passed along the axis of symmetry of the deposited spot. Macrosections were treated by $20 \%$ solution of nitric acid to reveal the penetration zone. Figure 1 shows samples of transverse macrosections of welds in surfacing with $4 \mathrm{~mm}$ electrode. Statistical processing of the results of measuring penetration zone parameters was followed by plotting approximation polynomial dependencies of its development in surfacing with electrodes of different diameters and arcing time (Figure 2).

Processing of experimental data was performed by the method of multiple regression analysis with screening variables at significance level $\alpha=0.03$ at the value of correlation coefficient $R=0.96-0.97$. Resulting mean deviation values were used to select third-degree polynomials, which showed quite good agreement with research results. Differentiation of the data of functional dependencies in time yielded models of the change of penetration zone growth rate up to its solidification within the used array of initial data. Investigation of models of penetration zone growth rate in each studied time interval showed that they can be generalized by a function of the following form: 

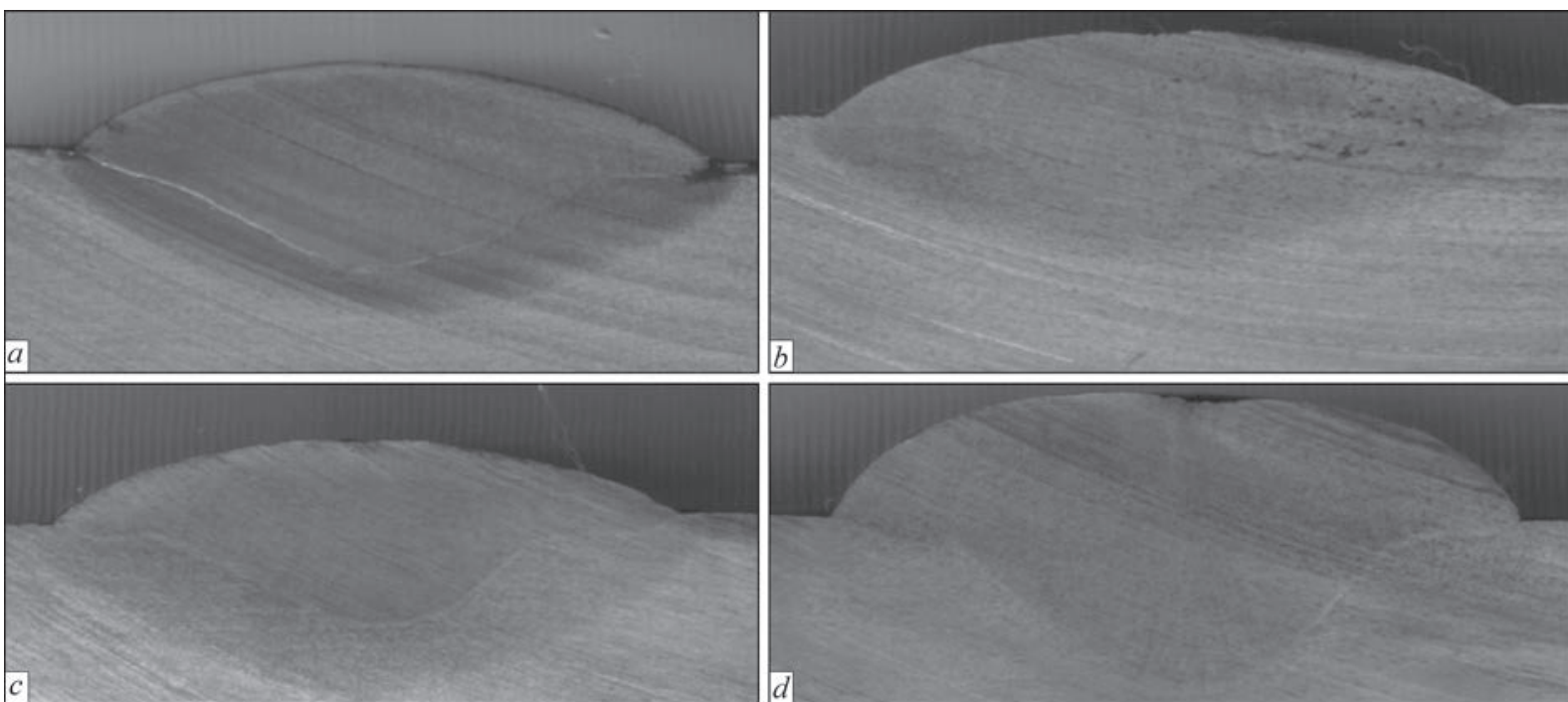

Figure 1. Cross-sections of surfaced samples at arcing time of: $a-2 ; b-5 ; c-7 ; d-10 \mathrm{~s}$ for $d_{\mathrm{el}}=4 \mathrm{~mm}, I_{\mathrm{s}}=600-650 \mathrm{~A}, v_{\mathrm{f}}=$ $=33 \cdot 10^{-3} \mathrm{~m} / \mathrm{s}, U_{\mathrm{a}}=30-32 \mathrm{~V}$
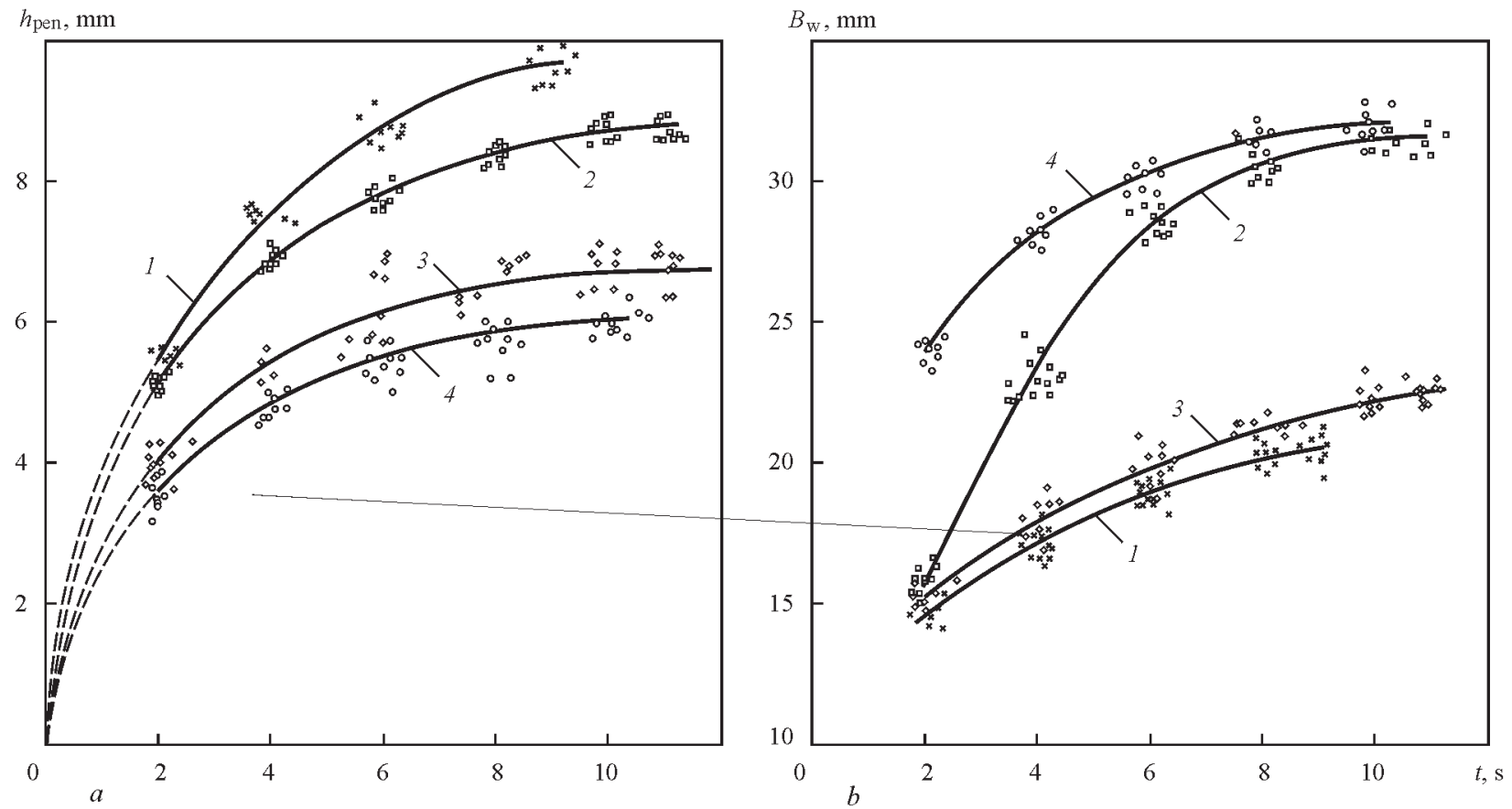

Figure 2. Dependence of penetration zone depth (a) and weld width (c) on arcing time $t: 1-d_{\mathrm{el}}=3 \mathrm{~mm}, I_{\mathrm{s}}=550 \mathrm{~A}, U_{\mathrm{a}}=30 \mathrm{~V} ; 2-$ $d_{\mathrm{el}}=4 \mathrm{~mm}, I_{\mathrm{s}}=650 \mathrm{~A}, U_{\mathrm{a}}=32 \mathrm{~V} ; 3-d_{\mathrm{el}}=4 \mathrm{~mm}, I_{\mathrm{s}}=550 \mathrm{~A}, U_{\mathrm{a}}=30 \mathrm{~V} ; 4-d_{\mathrm{el}}=5 \mathrm{~mm}, I_{\mathrm{s}}=550 \mathrm{~A}, U_{\mathrm{a}}=30 \mathrm{~V}$

$$
\bar{V}_{i}(\bar{x})=\left[\varepsilon_{1} \bar{X}^{2}+\varepsilon_{2} \exp (\bar{x})+\frac{\varepsilon_{3}}{\exp (\bar{x})}+\varepsilon_{4} \bar{X}+\varepsilon_{5}\right]^{-1},
$$

where $\overline{V_{i}}(\bar{x})$ is the relative rate of penetration zone growth for $i$-th interval; $\bar{x}$ is the relative coordinate of melting front point; $\varepsilon_{i}$ are the coefficients, which are the functional dependencies on technological parameters, where electrode feed rate and its diameter are the main independent variables.

Figure 3 shows the graphs of dependencies of relative rate of melting front propagation (according to the above dependence) in its formation cycle, divided into 10 equal time intervals $t_{i}$, where $i$ is the fraction of formation cycle. Figure 4 gives a comparison of calculated contours of penetration zone with the data of macrosection measurements for fixed values of their formation time. Analysis of obtained dependencies

Ranges of surfacing mode parameters variation

\begin{tabular}{|c|c|c|c|c|}
\hline \multirow{2}{*}{$d_{\mathrm{el}}, \mathrm{mm}$} & \multicolumn{4}{|c|}{ Parameters } \\
\cline { 2 - 5 } & $I_{\mathrm{s}}, \mathrm{A}$ & $v_{\mathrm{f}} \cdot 10^{-3}, \mathrm{~m} / \mathrm{s}$ & $l_{\mathrm{el}}, \mathrm{mm}$ & $U_{\mathrm{a}}, \mathrm{V}$ \\
\hline 1.6 & $180-300$ & $22-50$ & 20 & $26-30$ \\
\hline 2.0 & $210-390$ & $22-46$ & 25 & $26-30$ \\
\hline 3.0 & $280-550$ & $14-40$ & 30 & $28-32$ \\
\hline 3.5 & $300-600$ & $15-36$ & 30 & $30-34$ \\
\hline 4.0 & $400-700$ & $15-32$ & 40 & $30-34$ \\
\hline 5.0 & $500-800$ & $15-28$ & 45 & $30-36$ \\
\hline
\end{tabular}




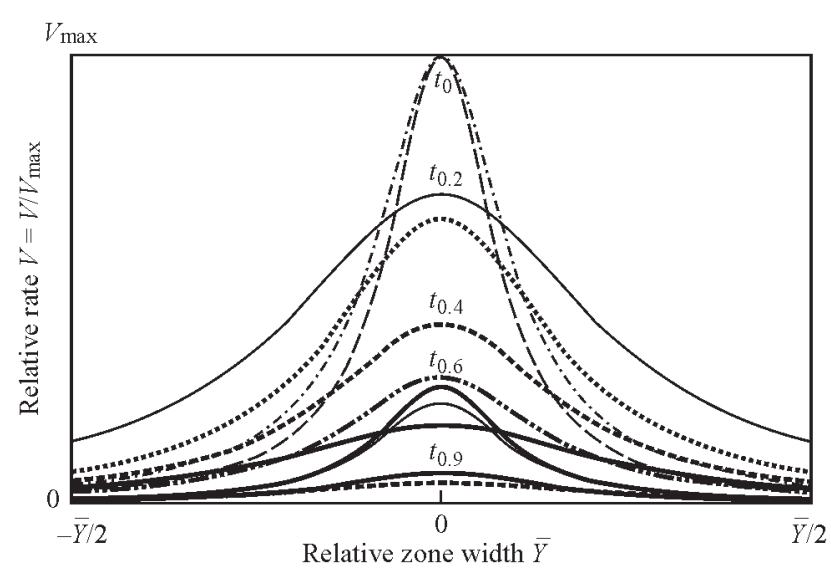

Figure 3. Dependencies of variation of relative rate of base metal melting front propagation

shows that after passing of $60-80 \%$ of zone growth time, the profile of the rates across pool cross-section is levelled, and the limits of the penetration zone become larger uniformly in all the directions, while the absolute value of the rate drops to zero. This is also confirmed by decrease of the value of discrepancy between the calculated and experimental data in this time interval (see Figure 4). Comparison of penetration zone profiles shows quite good agreement of calculated and experimental data, except for fusion boundary, that is associated with the model not allowing for force factors prevalent in this zone [7].

Extension of calculation results for stationary electrode to cases, when its movement speed is not equal to zero, involves application of functional dependence, relating the time of action of a stationary source in point $O$ with its movement speed. In the case of electrode movement, penetration maximum is at a certain distance from electrode axis, so that maximum penetration depth does not coincide with maximum reinforcement, the sum of which exactly makes up weld height $h$ after its solidification. This is rarely taken into account in calculation schemes, as weld pool front wall is represented as part of circumference with a center on electrode tip not only for a stationary, but also for a moving source. Thus, the value of welding speed is the parameter, determining the degree of weld pool asymmetry, which can be the main input parameter when solving problems of analysis based on non-deterministic models.

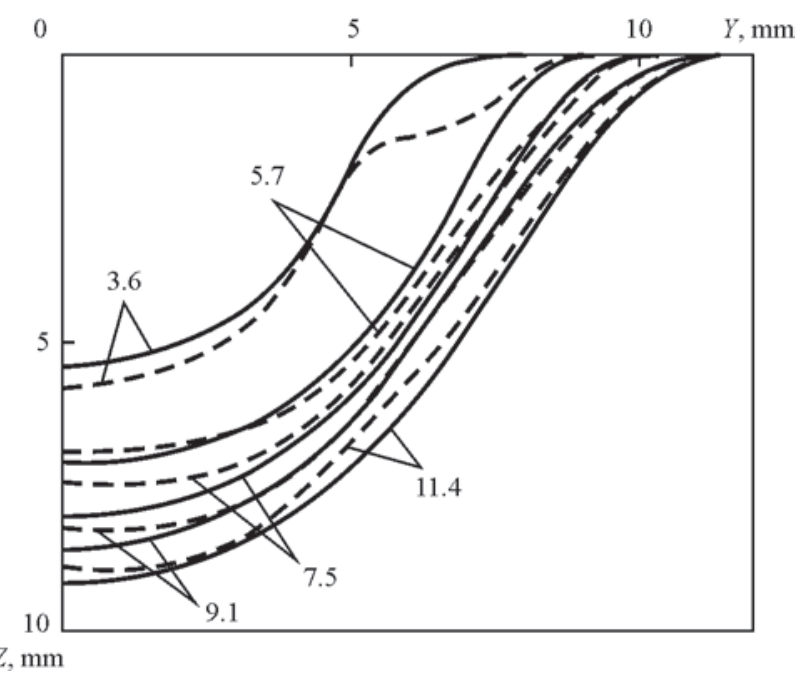

Figure 4. Calculated (solid curve) and experimental (dashed) contours of weld pool for $d_{\mathrm{el}}=4 \mathrm{~mm}, I_{\mathrm{s}}=650-700 \mathrm{~A}$, $v_{\mathrm{f}}=42 \cdot 10^{-3} \mathrm{~m} / \mathrm{s}, U_{\mathrm{a}}=30-32 \mathrm{~V}^{\mathrm{el}}$

Used as calculation scheme for stationary electrode (Figure 5) was the scheme with movement of quasistationary penetration zone of radius $R_{0}$, moving at speed $v_{\mathrm{w}}$ and recorded at moments of time $t_{i}$ (with step $\Delta$ ) [8].

Current position is determined by point $O^{\prime}$, in which a stationary penetration zone of radius $r_{i}$ forms. Step $\Delta_{i}$ determines time $t^{\prime}$, which is required for melting front to reach an area limited by radius $R_{0}$. It is assumed that after time step $t^{\prime}$ is over, solidification of weld pool of this contour, limited by isotherm $T_{\text {melt }}$, begins, and the source moves by step $\Delta$. Condition $\Delta^{\prime}=\Delta$ was used as process optimization condition, i.e. in this case, condition of maximum penetration zone at a given heat source power is fulfilled. In this case, minimum consumption of energy at specified weld parameters is ensured, i.e. to reduce penetration depth and proportion of base metal, optimization condition looks as $\Delta^{\prime}=\beta \Delta$, where $\beta$ is the coefficient, dependent on technological conditions [8].

Generalization of investigation results for the case of moving electrode leads to the conclusion that effective control of penetration zone formation in arc surfacing can be achieved by variation of two parameters: electrode feed rate and arc movement speed. So, Figure 6 gives the dependence of penetration zone depth on feed rate $v_{0}$ in automatic arc surfacing with wire electrode $\left(v_{\mathrm{w}}=15 \mathrm{~m} / \mathrm{h}\right)$. Macrosections for current

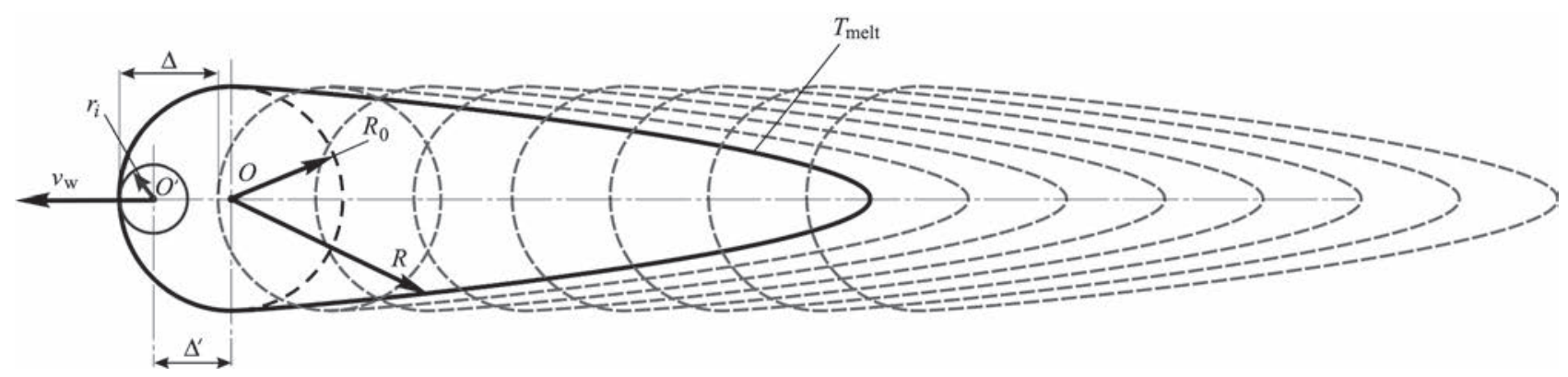

Figure 5. Calculation scheme for determination of weld pool dimensions in the case of electrode movement with welding speed 
values of 550 (1), 680 (2) and 750 (3) A are given for comparison (penetration zone is marked by a dashed line). The given data lead to the conclusion that the width of penetration zone is practically independent on $v_{0}$, and its certain reduction is, probably, related to a change of the size of effective heating zone at increase of feed rate (current) due to constriction of arc column and it penetrating deeper into base metal.

Developed models allow quantitative determination of the influence of variation of electrode feed rate by a certain law on penetration zone formation. In this case, the function of electrode speed variation is used as the argument of a system of equations for calculation of the rate of melting front propagation. This procedure was the base for creation of CAE-module for designing the surfacing technology (Figure 7), providing optimization of mode parameters, allowing for technology variant and applied welding consumables. The module includes a block for calculation of parameters of a deposited layer of a variable chemical composition in melting of two electrodes of different chemical composition in a common pool. Here, electrode feed rates are calculated, proceeding from the assigned law of variation of alloying element concen-

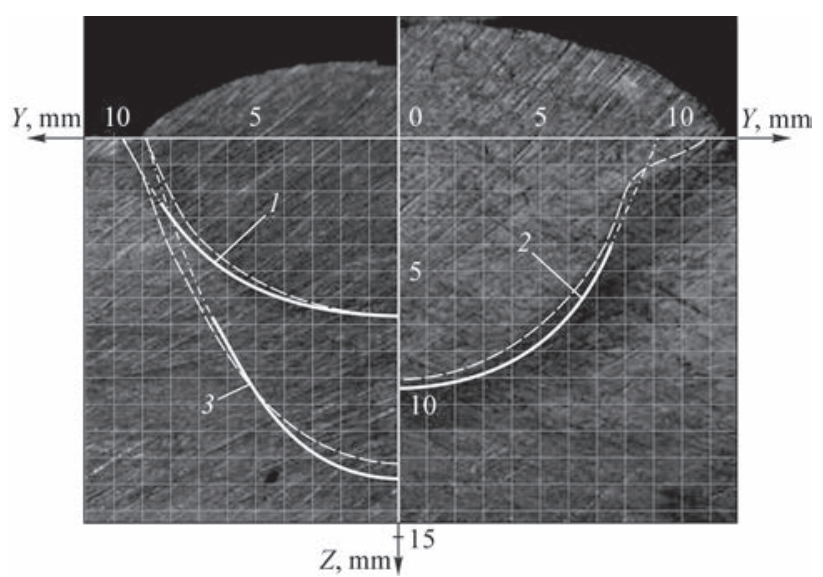

Figure 6. Dependence of penetration shape on feed rate of $4 \mathrm{~mm}$ wire electrode with $v \cdot 10^{-3}=25$ (1), 33 (2) and 44 (3) $\mathrm{mm} / \mathrm{s}$

tration in the transition section in the case of the pool quasistationary state.

A control system for the surfacing process (Figure 8) was developed, which uses calculated values of parameters of electrode feed rate and movement speed to generate control signals to the drives of surfacing unit equipment (Figure 9). Control system is a software-implemented phase position control circuit of the thyristors. «More-Less buttons» allow controlling

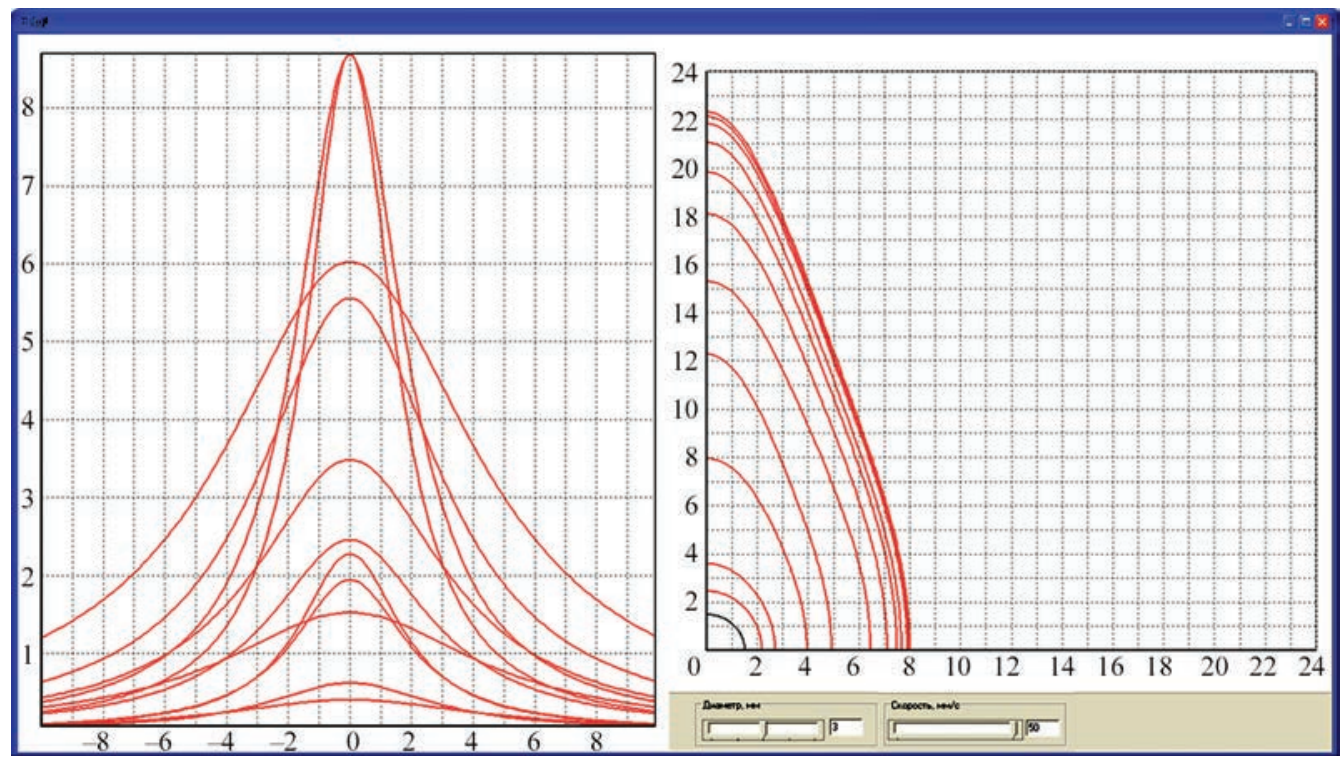

Figure 7. Module of penetration zone parameter calculation

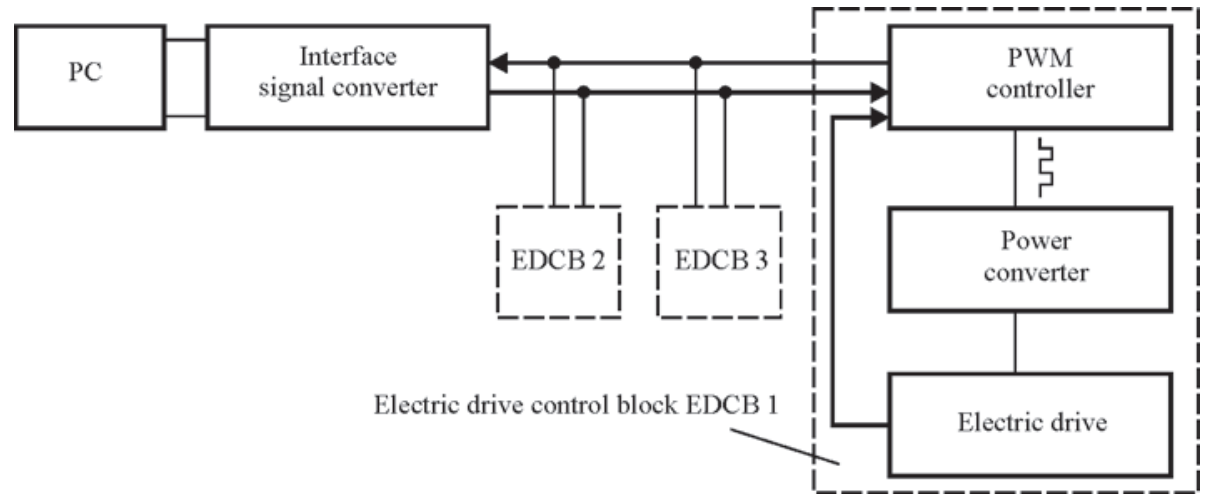

Figure 8. Block-diagram of surfacing process control system 


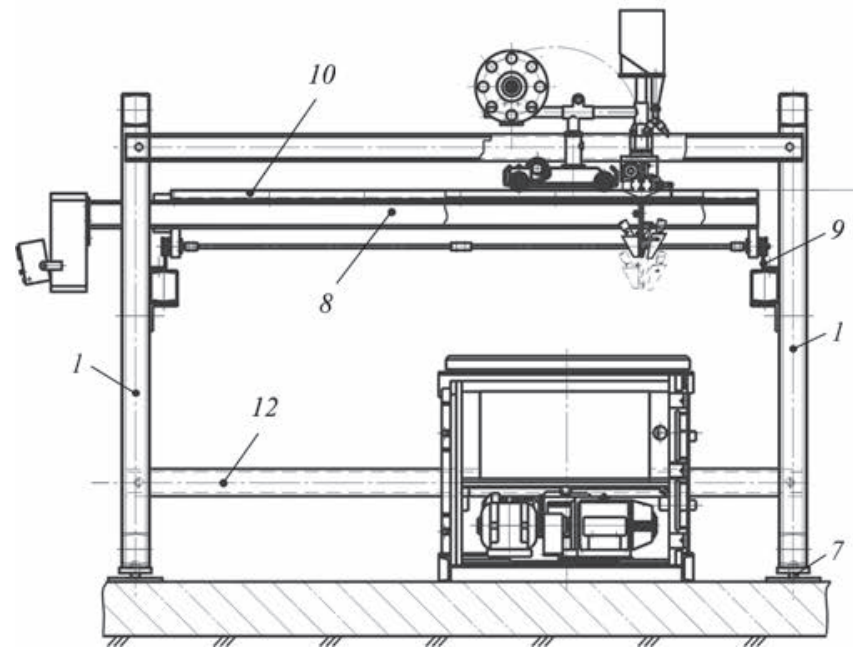

Figure 9. Schematic of surfacing unit (for designations see the text)

the moment of thyristor switching on. Control system status is saved in non-volatile processor memory, and at subsequent switching-on of the unit the last recorded status is read from the memory. Motors of feeding and movement of electrode and item are PWM-controlled, with time delay of motor switching-on being also implemented through software, and braking at switching off being performed through a relay.

Surfacing stand is of modular design (see Figure 9). Stand framework consists of four columns 1 on adjustable supports 7 and six longitudinal beams 12. Middle beams have a detachable connection with columns (bolted joints), and can be positioned with a certain step by height relative to surfaced item. These beams carry guides 9 for displacement of frame 8 with welding tractor 4 along the stand. Frame beams go beyond the stand framework dimensions, as they carry equipment for surfacing process control: cabinet 5 and removable control panel 6 , which is connected to the cabinet and manipulator 2 and to faceplate 3 by flexible cables.

All the design elements of the framework, as well as frame beams, have the same cross-section of boxlike structure, welded from two bent channel bars. Framework stability is provided by lateral braces made from channel bars.

As the lateral braces are removable, and moving frame guides are extendable, framework dimensions can be easily adjusted to suit the dimensions of the surfaced items. Transverse movement is performed by welding tractor drive. For this purpose guides 10 are mounted on frame 8 .

\section{Conclusions}

1. Influence of surfacing mode parameters on development of penetration zone in submerged-arc surfacing was studied for a stationary and moving arc. It is

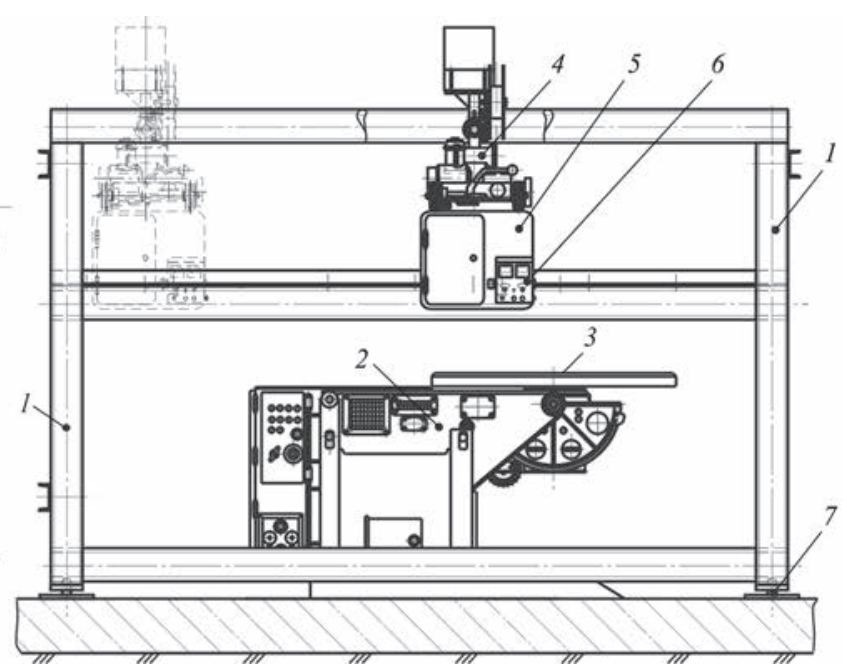

found that dependencies of the change of melting rates can be expressed by a generalized model (formula) for the entire interval of zone growth. For the case of an electrode, moving at welding speed, calculation by the developed model is performed with the condition of movement of a stationary penetration zone, recorded at moments $t_{i}$ with step $\Delta$ by movement coordinate.

2. Investigation results were used to develop mathematical models of penetration zone dynamics. Electrode feed rate and movement speed were taken as initial parameters. Software, equipment and control system were developed, which allow controlling deposited layer formation at the change of surfacing process parameters.

1. Paton, B.E., Lebedev, V.A. (2002) Analysis of technical and technological possibilities of electrode wire pulsed feed in arc welding and surfacing processes. Svarochn. Proizvodstvo, 2, 24-31.

2. Shaferovsky, V.A., Serenko, A.N. (2007) Method of submerged-arc welding of plate metal with programming of mode parameters. In: Proc. of 2nd Sci.-Techn. Seminar on Welding and Related Processes in Industry (17 April 2007, Kiev, Ukraine), 8-11.

3. Ivanov, V.P., Lavrova, E.V. (2014) Improving the efficiency of strip cladding by the control of electrode metal transfer. Appl. Mechanics and Materials, 682, 266-269.

4. Ivanov, V.P., Lavrova, E.V., Stepnova, Yu.A. (2015) Study of process of controlled mechanical transfer in surfacing with two strip electrode. Visnyk DonbasDMBA, 3, 102-107.

5. Kiyohara, M., Yamamoto, H.Y., Harada, S. (1979) Melting characteristics of a wire electrode in the MIG-welding of aluminium. In: Arc physics and weld pool behavior, Paper 26, 165-175.

6. Killing, R. (1991) Guenstige Strom-spannungsreihe beim MIG-/MAG-impulslichtbogenschweissen von Stahl und Aluminium unter verschiedenen Schutz Gazen. DVS Bericht, 131, 15-21.

7. Lu, S., Hidetoshi, F., Sugiyama, H. et al. (2003) Marangoni convection and welding penetration in A-TIG welding. Transact. of JWRI, 32(2), 79-82.

8. Ivanov, V.P. (2013) Increase in efficiency of arc surfacing process by control of welding pool hydrodynamics. Visnyk PryazovDTU, 26, 150-160.

Received 10.06.2016 